which the oscillation is not less than a given positive number form a closed set; the Du Bois-Reymond theorems on integration; the Sierpinski theorem; and others. The new concept lends itself readily to broad generalizations, and its simplicity suggests the possibility of advantageous use even in the usual theory.

10. Let $a_{i k}$ be the general element of the infinite determinant $D$ and assume the convergence of $\Sigma\left|a_{i k}\right|$. By comparison with an infinite product Professor Brenke obtains the following results, of which $(d)$ is a well-known theorem, from which also (a) might be derived: $(a) D$ converges absolutely to the value $0 ;(b)$ if the elements of any number of rows or columns of $D$ are replaced by quantities less in absolute value than a positive constant, the new determinant converges absolutely to 0 ; $(c)$ if all the elements $a_{i k}, i>k$, are replaced by quantities less in absolute value than 1, the new determinant converges absolutely; $(d)$ von Koch's “normal determinant" converges absolutely; (e) a normal determinant remains absolutely convergent if elements $a_{i k}$ are replaced as in $(c)$.

11. Professor Davis shows that if the difference between two complex vectors in space is $\delta_{1}+\sqrt{-1} \delta_{2}$ and if $k$ is $U V \delta_{1} \delta_{2}$, then the square of the distance between the complex vectors is $e^{\gamma \overline{-1 \theta}} T\left(\delta_{1}+k \delta_{2}\right) T\left(\delta_{1}-k \delta_{2}\right)$ where $\theta$ is the angle between $\delta_{1}+k \delta_{2}$ and $\delta_{1}-k \delta_{2}$. This is an extension to space of a formula of Laguerre.

O. D. KellogG, Secretary of the Section.

\title{
NOTE ON THE POTENTIAL AND THE ANTI- POTENTIAL GROUP OF A GIVEN GROUP.
}

BY PROFESSOR G. A. MILLER.

(Read before the American Mathematical Society at Chicago, December 29, 1914.)

$\S 1$. Introduction.

WiтH every regular substitution group there may be associated a conjugate substitution group on the same letters 
such that each of these two groups is composed of all the possible substitutions on these letters which are commutative with every substitution of the other group. This fundamental theorem was proved by C. Jordan in his doctor's dissertation* and the given groups are called conjoints in Jordan's Traité des substitutions, 1870, page 58. Somewhat later G. Frattini made a more detailed study of the properties of these two related groups, and called them the potential and the antipotential group respectively. $\dagger$

Some of the results obtained by Frattini seem to have received less attention than they deserve. This may be partly due to the facts that his developments are not always clear and that his erroneous statements tend to create a feeling of distrust on the part of the reader. One of these statements appears in the form of a theorem on page 168 of the article to which we have referred, and is so obviously incorrect that it appears curious to find that Netto repeated it in his review of the article in the Jahrbuch über die Fortschritte der Mathematik, volume 15, page 113.

This theorem affirms that if we form successive maximal subgroups, starting with a group $G$ and ending with identity, then the series of integers obtained by dividing the order of each of these groups by the order of the one which immediately follows it in the given series is an invariant of $G$, except possibly as regards the order in which the integers occur. The fact that this theorem is false can be readily seen by means of such a well known group as the icosahedral group. Equally obvious misstatements appear in the continuation of the same article, published in volume 18 of the same journal, but these errors also pass without mention by the reviewer in the Fortschritte.

In the present note we aim to prove in a simple manner a few of the results obtained by Frattini, and to extend some of his developments along certain lines. For brevity, the potential and the antipotential group will be represented by $G$ and $G^{\prime}$ respectively. We shall first establish the following elementary theorem:

The conjoint of every transitive constituent of a subgroup of $G$ is a transitive constituent of a subgroup of $G^{\prime}$, and vice versa. p. 39.

* C. Jordan, Thèse présentées a la Faculté des Sciences de Paris, 1860, † G. Frattini, Atti della R. Accademia dei Lincei. Memorie, vol. 14 (1883), p. 144. 
As a special case of this theorem it follows that every abelian transitive constituent of a subgroup of $G$ is a transitive constituent of a subgroup of $G^{\prime}$, and vice versa. As a special case of this special case it results that every cycle of every substitution of one of two conjoints is also a cycle of some substitution of the other.

To prove the theorem in question let $K$ represent the conjoint of a transitive constituent of a subgroup of order $k$ contained in $G$. This subgroup is clearly composed of all the substitutions of $G$ which transform $K$ into itself, and hence $K$ has $g / k$ conjugates under $G, g$ being the order of $G$. These conjugates are on distinct sets of letters. The $g / k$ conjugates of each substitution of $K$ may therefore be represented in the form of a single substitution which is invariant under $G$. Hence it is possible to establish a $(1,1)$ correspondence between the conjugates of $K$ so as to obtain a subgroup of order $k$ in $G^{\prime}$, and this subgroup involves as one of its transitive constituents the conjoint of an arbitrary transitive constituent of the arbitrary subgroup of $G$. Hence the theorem in question is established.

\section{§2. Conjugate Operators and Conjugate Subgroups.}

The above considerations give rise to a method for finding all the subgroups of $G$ which are conjugate with any one $H$ of its subgroups. If $H^{\prime}$ is any one of the subgroups of $G^{\prime}$ which has for one of its transitive constituents the conjoint of a transitive constituent of $H$, then it results that a necessary and sufficient condition that a given subgroup of $G$ is conjugate with $H$ is that it involves at least one transitive constituent which is the conjoint of some transitive constituent of $H^{\prime}$. That is,

$A$ necessary and sufficient condition that two subgroups of $G$ are conjugate under $G$ is that it is possible to select one transitive constituent from each of these two subgroups such that the conjoints of these two transitive constituents are transitive constituents of the same subgroup of $G^{\prime}$.

Each transitive constituent of a subgroup of $G$ is completely determined by the letters of this constituent. That is, if a subgroup of $G$ has a transitive constituent involving a certain set of letters, no other subgroup of $G$ has a transitive constituent on exactly the same set of letters. Hence the theorem 
stated at the end of the preceding paragraph may also be stated as follows:

$A$ necessary and sufficient condition that two subgroups of $G$ are conjugate is that it is possible to find a transitive constituent in each of these two subgroups such that these two transitive constituents involve separately the same letters as two transitive constituents of some subgroup of $G^{\prime}$.

As a special case of this theorem it results that a necessary and sufficient condition that a subgroup $H$ of $G$ is invariant is that $G^{\prime}$ involves a subgroup $H^{\prime}$ all of whose transitive constituents are the conjoints of the transitive constituents of $H$. As a special case of this special case it may be observed that

$A$ necessary and sufficient condition that a subgroup of $G$ is invariant is that the totality of the cycles in its substitutions is the same as that of a subgroup of $G^{\prime}$.

If we select two complete sets of conjugate subgroups belonging to $G$ and $G^{\prime}$ respectively, then either all the conjoints of the transitive constituents of the subgroups of the first set are transitive constituents of subgroups of the second set, or none of these conjoints are among the transitive constituents of the subgroups of the second set. In particular, two complete sets of conjugate substitutions belonging to $G$ and $G^{\prime}$ respectively either have all their cycles in common, or they have no transitive constituent in common. A necessary and sufficient condition that a transitive constituent of a subgroup of $G$ is also a transitive constituent of a subgroup of $G^{\prime}$ is that this subgroup is abelian. Hence it is possible to find all the conjugates of a subgroup by noting the transitive constituents of subgroups in the potential and antipotential representations.

\section{§3. Methods of Constructing the Potential and the Antipo- tential Group.}

To construct the potential and the antipotential group of a given abstract group $A$, Frattini proceeded practically as follows:* Let $1, s_{2}, s_{3}, \cdots, s_{g}$ represent the distinct operators of $A$ and construct the following two rectangular arrays:

* G. Frattini, loc. cit. 


\begin{tabular}{|c|c|c|c|}
\hline & $s_{2}$ & $s_{3}$ & $\cdots$ \\
\hline & $s_{2}^{2}$ & $s_{3} s_{2}$ & $\cdots$ \\
\hline & $s_{2} s_{3}$ & $s_{3}^{2}$ & - \\
\hline & . $\quad$. & - & $\cdot$ \\
\hline & . & • & • \\
\hline & $s_{2} s_{g}$ & $s_{3} s_{g}$ & \\
\hline
\end{tabular}

\begin{tabular}{|c|c|c|c|c|}
\hline 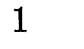 & $s_{2}$ & $s_{3}$ & $\cdots$ & $s_{g}$ \\
\hline$s_{2}^{-1}$ & 1 & $s_{2}^{-1} s_{3}$ & $\cdots$ & $s_{2}^{-1} s_{g}$ \\
\hline $3^{-1}$ & $s_{3}{ }^{-1} s_{2}$ & 1 & $\cdots$ & $s_{3}{ }^{-1} s_{g}$ \\
\hline - & $\cdot$ & $\cdot$ & $\cdot$ & • \\
\hline • & $\cdot$ & $\cdot$ & & . \\
\hline & $s_{g}-s_{2}$ & $s_{g}^{-1} s_{3}$ & & 1 \\
\hline
\end{tabular}

The substitutions which represent the permutations of the operators in the first array, if each row is compared with the first row, constitute the potential group $G$ of $A$, while those of the second array constitute the antipotential group $G^{\prime}$ of $A$. Both of these groups are simply isomorphic with $A$ and hence they are also simply isomorphic with each other.

Before the publication of this paper by Frattini it was customary to obtain the regular representation of a given substitution group by means of a function which changes its value for every substitution of the group, but during the same year in which Frattini's article appeared there appeared in the Mathematische Annalen, volume 22, an important article by Dyck entitled "Gruppentheoretische Studien II," in which the regular representation is derived in the same manner. In fact, this paper by Dyck and the paper by Frattini have many other things in common, but the more lucid presentation of these subjects in the former article has caused an undue neglect of the latter.

A simple isomorphism between $G$ and $G^{\prime}$ may be obtained by letting the substitutions obtained from each pair of corresponding rows in the given arrays correspond to each other. If the inverses of operators in the second array are replaced by the operators themselves, a simple isomorphism between $G$ and $G^{\prime}$ results. Moreover, it is clear that in the simple isomorphism between $G$ and $G^{\prime}$, which results directly from the given arrays, the first cycle of each substitution of $G$ is the inverse of the first cycle of the corresponding substitution of $G^{\prime}$.

This fact, combined with the fact that if the first two letters of each pair of corresponding substitutions of two simply isomorphic regular groups are the same then the corresponding substitutions are identical, leads to a very simple method for deriving $G$ from $G^{\prime}$ or vice versa. This method is embodied in the following theorem: 
The substitution of order 2 which transforms into their inverses each of the cycles of $G$ or $G^{\prime}$ which involve a given letter, and leaves this letter fixed, transforms $G$ and $G^{\prime}$ into each other.

Hence it is very easy to find either one of two conjoints when the other is given.

A substitution of order 2 which transforms the potential and the antipotential group into each other may be found from the fundamental group itself without the use of the regular representation. In fact, if $1, s_{2}, s_{3}, \cdots, s_{g}$ are the operators of the fundamental group and if we associate $g$ different letters with these operators, then cycles of these letters correspond to the various distinct powers of these operators. The substitution of order 2 in question may be obtained by finding the substitution which transforms each of these cycles whose order exceeds two into its inverse and omits the letter which corresponds to identity.

To illustrate this method, consider the group of order 8 on four letters, and represent its substitutions as follows:

$$
\begin{aligned}
& 1=A, \quad a c \cdot b d=B, \quad a c=C, \quad b d=D, \\
& a b c d=E, \quad a d c b=F, \quad a b \cdot c d=G, \quad a d \cdot b c=H .
\end{aligned}
$$

The only cycle on the capital letters whose order exceeds 2 is $E B F A$. Hence $E F$ transforms the potential and the antipotential group of the octic group into each other. That is, if the octic group is represented in the regular form on the given capital letters in accord with the usual process, then the transposition $E F$ will transform this regular group into its conjoint and vice versa. From the preceding developments there follows the theorem:

If each operator of any given group $A$ is represented by a distinct letter, then the substitution which transforms into their inverses the cycles representing the various distinct powers of all those operators whose orders exceed 2, and omits the letter corresponding to identity, transforms the conjoints of $A$ into each other, if these conjoints are written on these letters in the usual way.

When the group $A$ is an abelian group it is identical with its conjoint and hence it is transformed into itself by the substitution noted in the preceding theorem. In fact, in this case the substitution in question merely transforms into its inverse every operator of $A$ and hence it is contained 
in the holomorph of $A$. In general, this substitution transforms the holomorph of $G$ into itself; and when $G$ is nonabelian this substitution and the holomorph of $G$ generate the double holomorph of $G$. In regard to the given simple isomorphism between $G$ and $G^{\prime}$ it may be added that if we let every operator of any group correspond to its inverse we get a simple isomorphism only when the group is abelian; but if we multiply in one of these two corresponding groups on the right and in the other on the left there always results a certain kind of simple isomorphism. Moreover, it is easy to verify that if this kind of simple isomorphism can be established between two groups these groups must be actually simply isomorphic.

As regards the theorems relating to conjugate subgroups and invariant subgroups which were developed in $\S 2$, it should perhaps be emphasized that these theorems enable us to determine at a glance all the conjugates of a given subgroup, or of a given operator, provided the potential and the antipotential representation of the group are before us. Similarly they enable us to see at a glance what substitutions and what subgroups are invariant. On the other hand, it should be obsserved that the representation of a given group in the potential and the antipotential form is laborious, so that these theorems appear to be of more theoretical than practical interest.

\section{THE EQUATION OF PICARD-FUCHS FOR AN ALGEBRAIC SURFACE WITH ARBITRARY SINGULARITIES.}

BY DR. S. LEFSCHETZ.

(Read before the Southwestern Section of the American Mathematical Society, November 28, 1914.)

1. LET $F_{m}(x, y, z)=0$ be the equation of an algebraic surface of order $m$ with arbitrary singularities, the axes having an arbitrary position, and let

$$
\int \frac{P(x, y, z) d x}{F_{z}^{\prime}}
$$

be an abelian integral of the second kind attached to the 